\title{
A Form of the Euler Equations Preserving Potential Flow
}

\author{
P. L. Roe*and M. Rad ${ }^{\dagger}$ \\ W.M. Keck Foundation Laboratory for Computational.Fluid Dynamics \\ Department of Aerospace Engineering, University of Michigan, Ann Arbor, USA \\ and \\ Centre pour Mathematiques et leurs Applications \\ Ecole Normale Superieure de Cachan, France
}

\begin{abstract}
A new approach is taken to the computation of the elliptic part of the Euler equations. In each cell of an unstructured triangular grid, on which the solution is stored at the vertices, the residual is decomposed into purely elliptic and purely hyperbolic contributions. The elliptic part is minimised in a norm suggested by an earlier analysis of linearized potential flow. This choice of norm enables substantial simplification of the update procedure, for which an explicit formula is given. From the formula, it can be seen that the procedure is well-behaved in the transonic and lowMach number limits.
\end{abstract}

\section{Introduction}

This paper contributes further to the development of computational methods for the Euler equations (and eventually for other problems that share their structure) that reflect genuinely multidimensional physics. These methods aim to retain the benefits of a physical aproach to strongly discontinuous flows while avoiding the defects of upwind methods applied to almost incompressible flow. There has recently been considerable success in extending compressible flow codes to the low Mach number limit by means of matrix preconditioning. The present paper takes the approach of equation decomposition, which is a very closely related concept.

*Professor, University of Michigan, Visiting Professor, ENS Cachan, Fellow AIAA

${ }^{\dagger}$ Graduate Student, University of Michigan
The main contribution that we hope to make with this paper is to show that the decomposition, and the update procedure are sufficiently simple that explicit formulae can be given for them. This enables the singularities of the procedure, near sonic or stagnation points, to be displayed and percieved harmless.

\section{Forms of the Euler Equations}

Different aspects of the Euler equations are most readily expressed by choosing different sets of unknowns. For computing compressible flow, the most fundamental choice is probably the set of conserved quantities

$$
\mathbf{u}=(\rho, \rho \mathbf{v}, E)^{t}
$$

since a weak solution of this form of the equations captures shocks that satisfy the Rankine-Hugoniot conditions. Associated with these variables is the flux tensor

$$
\underline{\underline{\mathbf{F}}}=(\rho \mathbf{v}, \rho \mathbf{v} \otimes \mathbf{v}+p \underline{\underline{\mathbf{I}}}, \mathbf{v}(E+p))^{t}
$$

There is computational convenience in the parameter vector

$$
\mathbf{z}=\sqrt{\rho}(1, \mathbf{v}, h)^{t}
$$

where $h$ is the total specific enthalpy $(E+p) / \rho$, on account of the property that all components of $\mathbf{u}, \mathbf{\underline { \mathbf { F } }}$ are simply bilinear in terms of $\mathbf{z}$. This allows the construction of local linearizations having conservation properties, both in the one-dimensional ${ }^{1}$ and multidimensional ${ }^{2}$ cases. 
Also important, at least to the present approach, are what have been called ${ }^{3}$ the natural variables

$$
\mathbf{x}=(S, h, p, \mathbf{v} /|\mathbf{v}|)^{t}
$$

where $S$ is entropy, since it is uniquely in these variables that the Euler equations can be decoupled into maximally independent subsystems. ${ }^{4,5}$ This enables the essentially different hyperbolic and elliptic behaviour to be computed independently with the minimum of "crosstalk". In this paper we are parlicularly concerned with the solution of the elliptic subsystem.

\section{$\underline{\text { Fluctuation Splitting }}$}

In this approach ${ }^{6,7}$, the computational domain is divided into elements in an unstructured way, with the unknowns stored at its vertices. Iteration toward a steady solution from an in intial guess takes place by computing an average residual for each cell (the fluctuation $\phi^{T}$ in element $T$ ), and then changing the current solution at each node of the cell $T$ by an amount proportional to $\phi^{T}$;

$$
\delta \mathbf{u}_{j}=\omega \alpha_{j}^{T} \phi^{T} \quad(\text { if node } j \text { belongs to cell } T \text { ) }
$$

where $\omega$ is a relaxation factor and the weight $\alpha_{j}^{T}$ is a matrix to be determined.

Our aim is to create a method of this kind that exploits the different properties of the different sets of unknowns. We represent the solution in terms of the parameter vector, and find the conservative residual by integrating over the cell (of area $A^{T}$ )

$$
\phi_{\mathbf{u}}^{T}=C \partial_{x} \mathbf{z}+D \partial_{y} \mathbf{z} \frac{1}{A^{T}} \oint_{\partial T} C \mathbf{x} d y-D \mathbf{x} d x
$$

where the matrices $C=\partial \underline{\underline{\mathbf{F}}} / \partial \mathbf{z}$ and $C=\partial \underline{\underline{\mathbf{F}}} / \partial \mathbf{z}$ are locally constant because of the quadratic property ${ }^{2}$. Our aim is then to reduce this residual in some suitable norm. The norm is chosen by splitting the residual into its elliptic and hyperbolic parts.

Conceptually this is a sophisticated approach, but the fact that the final result emerges as the concatenation of a long chain of tranformations makes it seem costly. In fact, this would not be the proper approach, for reasons having nothing to do with cost.
Many of the transformations are singular, at $M=0$ or $M=1$ and although we expect that these singularities would cancel out in analysis, they are unlikely to do so numerically. Therefore we carry out the complete chain analytically, and find that its final form is computationally tractable and indeed rather simple if the correct norm is chosen.

\section{The Update Malrix}

This gives a convenient way to think about Fluctuation-Splitting schemes. Let the initial state of a cell (in two dimensions) whose vertices are $(a, b, c)$ be represented by the twelve scalar quantities

$$
\mathbf{W}^{n}=\left(\mathbf{w}_{a}, \mathbf{w}_{b}, \mathbf{w}_{c}\right)
$$

where $\mathbf{w}$ is whatever quantity we have decided to store at the vertices The update can be represented as a matrix multiplication

$$
\mathbf{W}^{n+1}-\mathbf{W}^{n}=\omega U \mathbf{W}^{n}
$$

where $U$ is some matrix that is constant within the cell and $\omega$ is a relaxation constant.

For example, suppose we intend to update the solution by a steepest-descent minimisation of

$$
\Phi=\sum_{T}\left(\phi^{T}\right)^{\iota} Q^{T}\left(\phi^{T}\right)
$$

where $Q$ is some symmetric matrix representing a local norm. If we write $Q=P^{t} P$ this is equivalent to an $L_{2}$ minimization of $P \phi^{T}$, which is some weighted combination of the residuals. We will treat $\phi^{T}$ as a linear function $R^{T} \mathbf{W}^{T}$ of the vertex values because it arises from a linear process to find the local derivatives, followed by multiplication by matrices that are frozen during the update. In general $R$ is a rectangular matrix formed from three $4 \times 4$ blocks (see the examples below). Hence the quantity to be minimized is

$$
\sum_{T}\left(\mathbf{W}^{t} R^{t}\right)^{T}\left(P^{t} P\right)^{T}(R \mathbf{W})^{T}
$$

and the gradient of this is

$$
\frac{\partial \Phi}{\partial \mathbf{w}_{j}}=2 \sum_{T_{j}}\left(R^{t} P^{t} P R\right)^{T} \mathbf{w}_{j}
$$


and so the update procedure within each triangle

$$
\mathbf{W}^{n+1}-\mathbf{W}^{n}=-\omega\left(R^{t} P^{t} P R\right)^{T} \mathbf{W}
$$

where $\omega$ is some global constant, implements the required procedure. In this case $U$ is the symmetric matrix $\left(R^{t} P^{t} P R\right)^{T}$.

If we had proposed some other method than steepest descent for solving the least-squares system, the update matrix would be different. However, the right-hand factors $(P R)^{T} \mathbf{W}$ which represent the weighted residual, would be exactly the same. These terms give the properly weighted elliptic part of the residual, and might be supposed rather complicated, but are actually very simple. The expression is given later as (8).

For the sake of definiteness we continue here with the least-squares procedure, whose properties will depend on the matrix $Q$ that defines the norm, or equivalently on the matrix $P$ which forms some linear combination of the scalar residual, and also on the matrix $R$ that defines the residuals. In fact we will only apply this technique to the elliptic part of the Euler equations since the hyperbolic parts are better handled by asymmetric, upwind, methods.

We design the procedure by first. considering the nonconservative case where the stored variables are $x$. The Euler equations for this case are $^{3}$

$$
\begin{aligned}
\left(1-M^{2}\right) p_{s}-\rho q^{2} \theta_{n} & =0 \\
\rho q^{2} \theta_{s}+p_{n} & =0 \\
\partial_{s} S & =0 \\
\partial_{s} h & =0
\end{aligned}
$$

The four residuals so defined may be called $\phi_{\mathbf{X}}$ where

$$
\phi_{\mathbf{x}}=\sum_{j=a, b, c} R_{j} \mathbf{x}_{j}
$$

where, using standard methods to evaluate the derivatives,

$$
R_{j}=\frac{1}{A^{T}}\left[\begin{array}{cccc}
\left(1-M^{2}\right) u_{n_{j}} & u_{s_{j}} & 0 & 0 \\
-u_{s_{j}} & u_{n_{j}} & 0 & 0 \\
0 & 0 & u_{n_{j}} & 0 \\
0 & 0 & 0 & u_{n_{j}}
\end{array}\right]
$$

where $u_{n_{j}}=\left[u\left(\Delta_{y}\right)_{j}-v\left(\Delta_{x}\right)_{j}\right]$ and $u_{s_{j}}=\left[v\left(\Delta_{y}\right)_{j}+\right.$ $\left.u\left(\Delta_{x}\right)_{j}\right]$ with $\left(\left(\Delta_{x}\right)_{j},\left(\Delta_{y}\right)_{j}\right)$ the vector representing the side opposite vertex $j$. The first two components of this vector comprise the elliptic part of the problem, if $M<1$ so the quantity to be minimized is

$$
\mathcal{D} \sum_{j=a, b, c} R_{j} \mathbf{x}_{j} \text { where } \mathcal{D}=\operatorname{diag}\left(d_{1}, d_{2}, 0,0\right)
$$

and the update matrix is

$$
U=\left(\sum_{j=a, b, c} R_{j}\right)^{t} \mathcal{D}^{2}\left(\sum_{j=a, b, c} R_{j}\right)
$$

The outcome still contains $p_{1}, p_{2}$ as free parameters, and there is a special choice of their ratio that brings about considerable simplification. This was anticipated on the strength of analysing linearised potential llow $^{8}$, where it was found that minimizing in a norm

$$
\begin{gathered}
\text { (continuity equation) }^{2} \\
+\left|1-M^{2}\right|{\text { (vorticity equation })^{2}}^{2}
\end{gathered}
$$

led to a method almost completely equivalent to applying the standard Galerkin finite-element to the Prandtl-Glauert equation ("stretched" Laplace equation) satisfied by each velocity component. It also provides for a smooth transition from the subsonic to the supersonic case. Thus, we choose $d_{1}=1, d_{2}=$ $\sqrt{1-M^{2}}$. Then one block of the update matrix becomes

$$
U_{i j}=\left[\begin{array}{cccc}
\left(1-M^{2}\right) a_{i j} & \rho q^{2} u_{n_{i}} u_{s_{j}} & 0 & 0 \\
-\rho q^{2} u_{n_{i}} u_{s_{j}} & \rho^{2} q^{4} a_{i_{j}} & 0 & 0 \\
0 & 0 & 0 & 0 \\
0 & 0 & 0 * & 0
\end{array}\right]
$$

where $a_{i j}=\left(1-M^{2}\right) u_{n_{i}} u_{n_{j}}+u_{s_{i}} u_{s_{j}}$. The offdiagonal terms are zero if $i=j$ and if the relaxation factor is taken inversely proportional to $\rho q^{2}$ it can be shown that they cancel around any interior vertex.

Turning now to the case where the stored variables are chosen as $\mathrm{z}$ (to ensure conservation) we continue to minimize in the same norm. That is to say, the quantity to be minimized is now defined by

$$
\phi_{P}^{T}=\sum_{j=a, b, c} R_{j} \frac{\partial \mathbf{x}}{\partial \mathbf{z}} \mathbf{z}_{j}
$$


It is important to note that this contains the conservative residual

$$
\sum_{j}\left[C\left(\Delta_{y}\right)_{j}-D\left(\Delta_{x}\right)_{j}\right) \mathbf{z}_{j}
$$

as a factor, because both are bilinear functions of $\mathrm{Z}$ that vanish simultaneously. The update matrix becomes

$$
U=\left(\sum_{j=a, b, c} R_{j} \frac{\partial \mathbf{x}}{\partial \mathbf{z}}\right)^{t} \mathcal{D}^{2}\left(\sum_{j=a, b, c} R_{j} \frac{\partial \mathbf{x}}{\partial \mathbf{z}}\right)
$$

\section{The Structure of $U$}

The update matrix $U$ actually enjoys considerable structure which can be demonstrated by exhibiting just one of the blocks;

$$
\frac{\partial\left(\phi_{P}^{T}\right)}{\partial \mathbf{z}_{a}}=\sqrt{1-M^{2}} u_{n_{a}} N+u_{s_{a}} S
$$

where

$$
\begin{aligned}
& N=\left[\begin{array}{cccc}
-k z_{4} & k z_{2} & k z_{3} & -k z_{1} \\
0 & z_{3} & -z_{2} & 0 \\
0 & 0 & 0 & 0 \\
0 & 0 & 0 & 0
\end{array}\right], \\
& S=\left[\begin{array}{cccc}
0 & -z_{3} & z_{2} & 0 \\
-k z_{4} & k z_{2} & k z_{3} & -k z_{1} \\
0 & 0 & 0 & 0 \\
0 & 0 & 0 & 0
\end{array}\right]
\end{aligned}
$$

and

$$
k=\frac{\gamma-1}{\gamma} \sqrt{1-M^{2}}
$$

Hence that part of the update matrix giving the effect of node $i$ on node $j$ is

$$
\begin{array}{r}
U_{i, j}=\left[\sqrt{1-M^{2}} u_{n_{i}} N+u_{s_{i}} S\right]^{t} \times \\
\left.\left[\sqrt{1-M^{2}} u_{n_{j}} N+u_{s_{j}} S\right)\right]
\end{array}
$$

At this point it turns out that $N^{t} N=S^{t} S$ and $S^{t} N+$ $N^{t} S=0$ so the update becomes

$$
\begin{aligned}
U_{i, j}= & {\left[\left(1-M^{2}\right) u_{s_{i}} u_{s_{j}}+u_{n_{i}} u_{n_{j}}\right]\left(S^{t} S\right) } \\
& +\sqrt{1-M^{2}}\left[u_{s_{i}} u_{n_{j}}-u_{s_{j}} u_{n_{i}}\right]\left(S^{t} N\right)
\end{aligned}
$$

where

$$
\begin{aligned}
S^{t} S=k^{2} & {\left[\begin{array}{cccc}
z_{4}^{2} & -z_{2} z_{4} & -z_{3} z_{4} & z_{1} z_{4} \\
-z_{2} z_{4} & z_{2}^{2} & z_{2} z_{3} & -z_{1} z_{2} \\
-z_{3} z_{4} & z_{2} z_{3} & z_{3}^{2} & -z_{1} z_{3} \\
z_{1} z_{4} & -z_{1} z_{2} & -z_{1} z_{3} & z_{1}^{2}
\end{array}\right] } \\
& +\left[\begin{array}{cccc}
0 & 0 & 0 & 0 \\
0 & z_{3}^{2} & -z_{2} z_{3} & 0 \\
0 & -z_{2} z_{3} & z_{2}^{2} & 0 \\
0 & 0 & 0 & 0
\end{array}\right]
\end{aligned}
$$

and

$$
S^{t} N=k\left[\begin{array}{cccc}
0 & z_{3} z_{4} & -z_{2} z_{4} & 0 \\
-z_{3} z_{4} & 0 & z_{2}^{2}+z_{3}^{2} & -z_{1} z_{3} \\
z_{2} z_{4} & -z_{2}^{2}-z_{3}^{2} & 0 & z_{1} z_{2} \\
0 & z_{1} z_{3} & -z_{1} z_{2} & 0
\end{array}\right]
$$

Defining vectors $\mathbf{a}=\left(z_{4},-z_{2},-z_{3}, z_{1}\right)^{t}$, and $\mathbf{b}=$ $\left(0, z_{3},-z_{2}, 0\right)^{t}$ we have the simple expressions

$$
\begin{gathered}
S^{t} S=k^{2} \mathbf{a}^{t} \mathbf{a}+\mathbf{b}^{t} \mathbf{b} \\
S^{t} N=\mathbf{a}^{t} \mathbf{b}-\mathbf{b}^{t} \mathbf{a}
\end{gathered}
$$

It is interesting that each block of $U$ has the same two-dimensional nullspace, spanned by

$$
\left[\begin{array}{c}
z_{1} \\
0 \\
0 \\
-z_{4}
\end{array}\right],\left[\begin{array}{c}
0 \\
z_{1} z_{2} \\
z_{1} z_{3} \\
z_{2}^{2}+z_{3}^{2}
\end{array}\right]
$$

The first of these shows that constant-enthalpy solutions are accepted. The second shows after some algebra that a scaling of the velocities and the sound speed by the same amount also leaves the solution unchanged.

\section{Transonic Transition}

It is instructive to collect the last result, using the definition of $k$, as

$$
\begin{aligned}
U_{i, j}= & \frac{(\gamma-1)^{2}\left(1-M^{2}\right)^{2}}{\gamma^{2}}\left(u_{n_{i}} u_{n_{j}}\right) \mathbf{a}^{t} \mathbf{a} \\
& +\frac{(\gamma-1)^{2}\left(1-M^{2}\right)}{\gamma^{2}}\left(u_{s_{i}} u_{s_{j}}\right) \mathbf{a}^{t} \mathbf{a}
\end{aligned}
$$




$$
\begin{aligned}
& +\frac{(\gamma-1)\left(1-M^{2}\right)}{\gamma} \times \\
& \left(u_{s_{i}} u_{n_{j}}-u_{s_{j}} u_{n_{i}}\right)\left(\mathbf{a}^{t} \mathbf{b}-\mathbf{b}^{t} \mathbf{a}\right) \\
& +\left(1-M^{2}\right)\left(u_{n_{i}} u_{n_{j}}\right) \mathbf{b}^{t} \mathbf{b} \\
& +\left(u_{s_{i}} u_{s_{i}}\right) \mathbf{b}^{t} \mathbf{b}
\end{aligned}
$$

The terms on the third line are the ones that can be ncglected in the constant-coefficient case; it is not yet clear whether they can be neglected here.

The conclusion of ${ }^{8}$ is that in the supersonic case, the appropriate norm is obtained by taking $d_{1}=$ $1, d_{2}=\sqrt{M^{2}-1}$ which causes the update of the characteristic variables $\sqrt{M^{2}-1} p \pm \rho q^{2} \theta$ to become decoupled from each other. Repeating the above analysis in the new norm leads to the following update matrix

$$
\begin{aligned}
U_{i, j}= & \frac{(\gamma-1)^{2}\left(M^{2}-1\right)^{2}}{\gamma^{2}}\left(u_{n_{i}} u_{n_{j}}\right) \mathbf{a}^{t} \mathbf{a} \\
& +\frac{(\gamma-1)^{2}\left(M^{2}-1\right)}{\gamma^{2}}\left(u_{s_{i}} u_{s_{j}}\right) \mathbf{a}^{t} \mathbf{a} \\
& +\frac{(\gamma-1)\left(M^{2}-1\right)}{\gamma} \times \\
& \left(u_{s_{i}} u_{n_{j}}+u_{s_{j}} u_{n_{i}}\right)\left(\mathbf{a}^{t} \mathbf{b}+\mathbf{b}^{t} \mathbf{a}\right) \\
& \left.+\left(M^{2}-1\right)\right)\left(u_{n_{i}} u_{n_{j}}\right) \mathbf{b}^{t} \mathbf{b} \\
& +\left(u_{s_{i}} u_{s_{j}}\right) \mathbf{b}^{t} \mathbf{b}
\end{aligned}
$$

What happens is that the first and last terms remain unaltered, the second and fourth terms change sign, but the third term is completely changed. The supersonic version does not even approximatcly cancel when summed round an interior vertex. However, the transition from slightly subsonic to slightly supersonic flow takes place smoothly, because all the changes happen in terms that vanish at $M=1$. We repeat that the use of a least-squares method is inappropriate when the flow becomes hyperbolic, but the analysis does demonstrate that at least the transition would be smooth. That is a property that should be retained.

\section{Stagnations Points and Incompressible Flow}

We notc that there are no singularities involved at $M=0$. We also note that when $M=0$ the update matrix simplifies to

$$
U_{i j}=\left(u_{s_{i}} u_{s_{j}}+\left(u_{n_{i}} u_{n_{j}}\right)\left[\frac{\gamma-1)^{2}}{\gamma^{2}} \mathbf{a}^{t} \mathbf{a}+\mathbf{b}^{a} \mathbf{b}\right]\right.
$$

which does not seem to reveal any computational difficulties.

\section{Computational Examples}

The three examples presented are in fact of potential flow, computed with the nonconservative version of the code. The first example is that of barely subcritical flow past a circular cylinder, It demonstrates the excellent behaviour of the least-squares technique right up to the stagnation points, even though the method used is applied not to the actual potential equation, but to the first-order system.

The second example is that of an ellipse placed broadside to the flow, merely to show that even in this case a very high degree of symmetry is maintained.

The third example is a symmetrical airfoil at zero incidence, and now the least-squares method is used only in the subsonic region. That is to say, everywhere in the first case, but only outside of

the supercritical zone in the second case. Where a supercritical region exists, the hyperbolic part of the problem is handled using the PSI advection scheme. No special procedure was used to match the calculations across the sonic

line or across the shock.

\section{Concluding Remarks}

The present contribution is not yet intended to provide a practical recipe for solving the Euler equations. The main defect is that steepest descent is, by itself, a very slow way to solve nonlinear equations. However, it forms the starting point for many other (mainly Newton-like) methods that are extremely efficient, and which have the same fixed points. It also remains to be proved that an analogous method will work in three dimensions. There is no problem formally. All the formulae given here cxtend straightforwardly. The issue is whether the streamwise vorticity that becomes coupled to the potential flow in three dimensions ${ }^{5}$ will be well captured. 


\section{References}

1. P. L. Roe, Approximate Riemann solvers, parameter vectors and difference schemes, J. Comput. Phys., 43, pp357-372, 1981.

2. H. Deconinck, P. L. Roe, R. J, Struijs, A multidimensional generalisation of Roe's flux difference splitter for the Euler equations, Computers and Flu$i d s$, 22, p215, 1993.

3. W. Hayes, R. F. Probstein Hypersonic Flow Theory, Academic Press, 1963.

4. P.L. Roe, L. M. Mesaros, Solving steady mixed conservation laws by elliptic/hyperbolic splitting, 13th International Conference on Numerical Methods in Fluid Dynamics, Monterey, July, 1996.

5. P.L. Roe, E.Turkel, The quest for diagonalization of differential systems, Workshop on Barriers and Challenges in Computational Fluid Dynamics, NASA Langley, August, 1996, Kluwer, 1998. pub by Springer, 1997.

6. P.L.Roe, Multidimensional upwinding, motivation and concepts, von Karman Institute Lecture Series 1994-04

7. H. Deconinck, H. Paillèrre, P.L. Roe, Conservative upwind residual-distribution schemes based on the steady characteristics of the Euler equations. AIAA CFD Meeting, San Diego, June, 1995.

8. P. L. Roe, Compounded of Many Simples, reflection on the role of model problems in CFD, Workshop on Barriers and Challenges in Computational Fluid Dynamics, NASA Langley, August, 1996, Kluwer, 1998. pub by Springer, 1997.
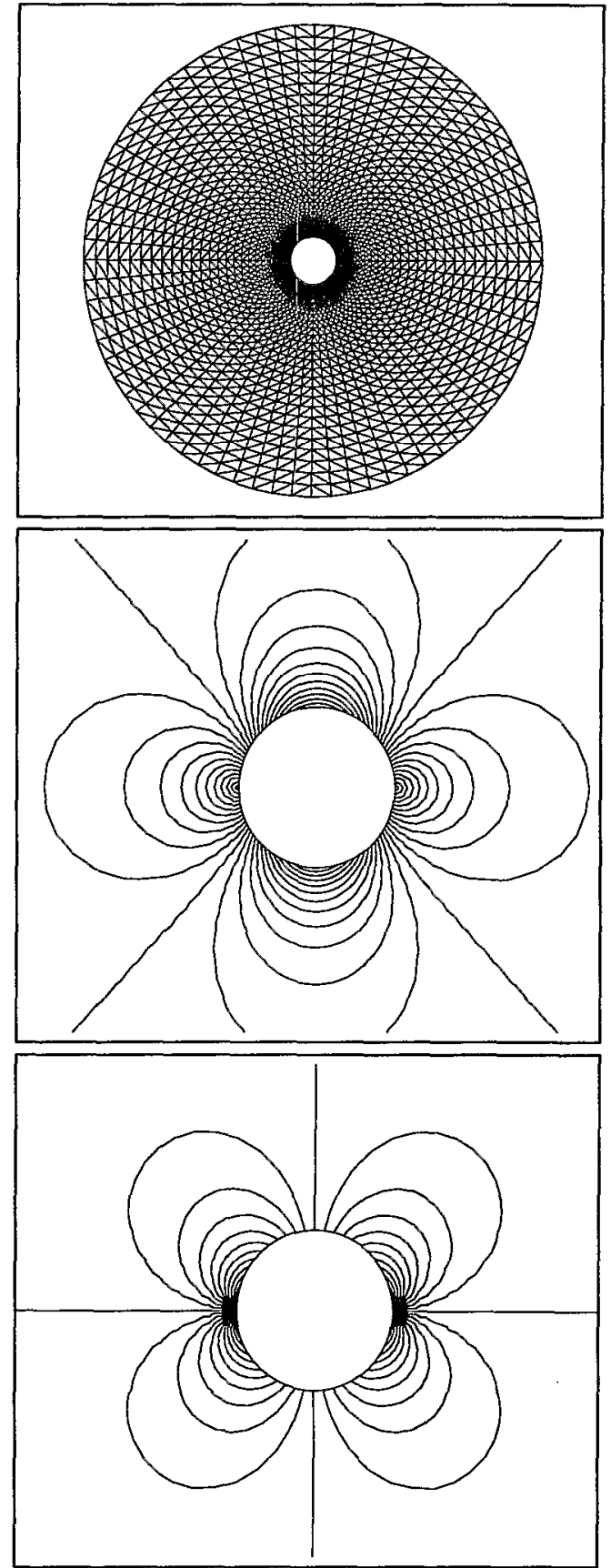

Figure 1: Subcritical cylinder $\left(M_{\infty}=\right.$ 0.38 ), grid, Mach contours and flow angle isolines. The challenge in this test case comes from the two stagnation points and in maintaining perfect symmetry with respect to the horizontal and vertical axes. 

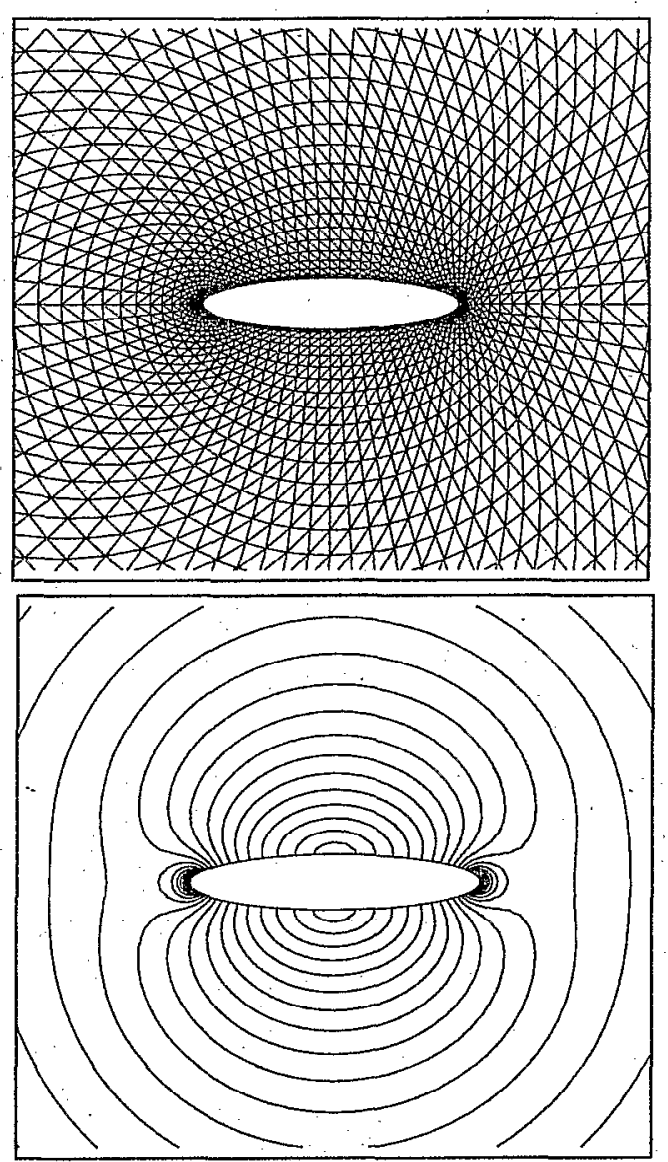

Figure 2: Subcritical ellipse $\left(M_{\infty}=0.1\right)$ at $90^{\circ}$ angle of attack, Mach contours. top/bottom and left/right symmetry are the result of the very low level of numerical vorticity generated by the least squares scheme.
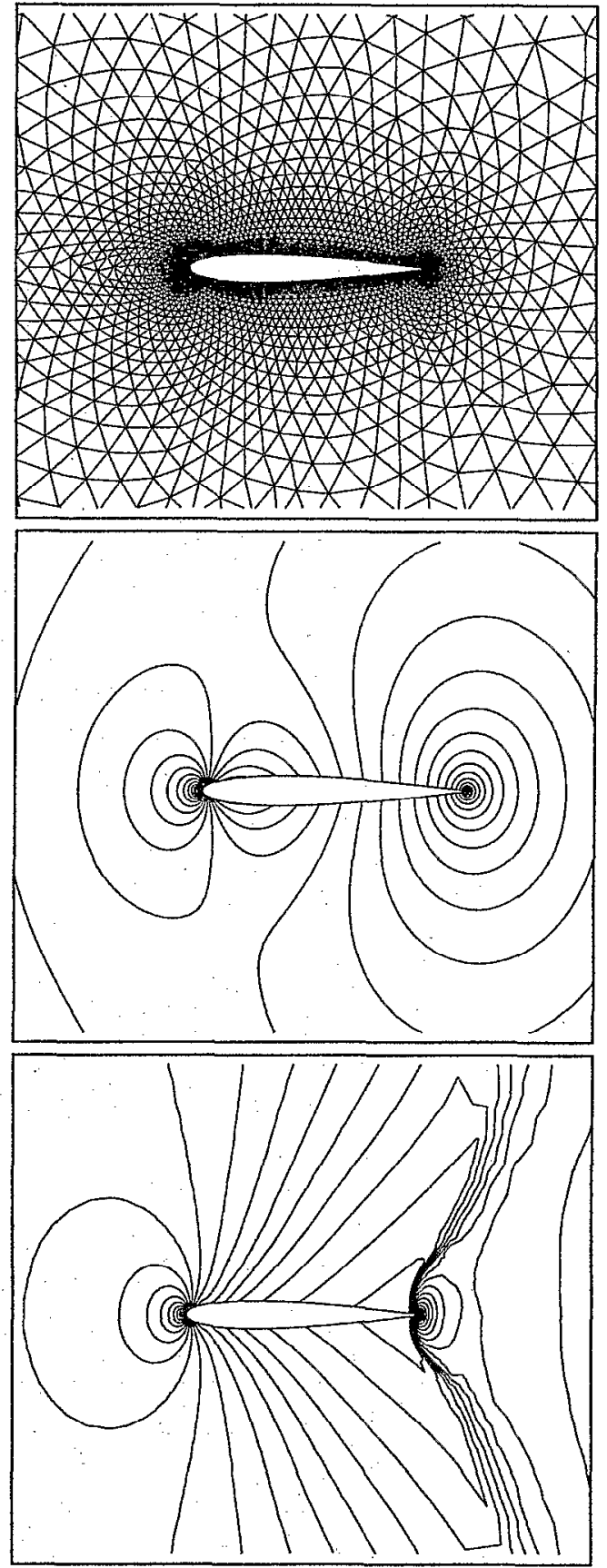

Figure 3: Subsonic $\left(M_{\infty}=0.36\right)$ and transonic $\left(M_{\infty}=0.85\right)$ NACA 0012, Mach contours. The purely subsonic case is solved using the least squares approach while the transonic case is calculated by combining least squares (subsonic region) and fluctuation splitting (supersonic region). 